\title{
On the Structure of Secret Key Exchange Protocols
}

\author{
Mihir Bellare * Lenore Cowen $^{\dagger} \quad$ Shafi
MIT Laboratory for Computer Science
545 Technology Square \\ Cambridge, MA 02139
}

\section{Introduction}

Modern cryptography is fundamentally concerned with the problem of secure private communication. Suppose two parties, Alice and Bob, wish to communicate privately over a public channel (for instance, a telephone line with an eavesdropper). If Alice and Bob are able to meet, privately, beforehand, and agree on some common secret key, then it becomes easy for them to achieve such private communication. But Alice and Bob might not be able to first meet in private and agree on a key. In this case, we ask under what assumptions they can still agree on a common secret key, where their conversation is conducted entirely in public.

A Secret Key Exchange is a protocol where Alice and Bob, having no secret information in common to start, are able to agree on a common secret key, conversing over a public channel. Secret Key Exchange is of course trivial if trapdoor permutations exist. However, there is no known implementation based on a weaker general assumption.

Recently [Impaggliazo, Rudich STOC 89] showed that any "natural" proof that secret key exchange was possible with one way permutations would imply a proof that $P \neq N P$. In this paper we look at the other side of the coin. We ask, what are the conditions necessary for Secret Key Exchange and what is its power to yield other important primitives? We define the Matrix of Conversations for any Secret Key Exchange protocol and show it must have strong structural properties. The results we obtain follow directly from our anaylsis of monochromatic blocks of this matrix.

In particular, we show that the existence of a Secret Key Exchange protocol implies that a one-way function exists, (as a corollary, we immediately get that Oblivious transfer implies the existence of one-way functions), and that Secret Key Exchange implies the existence of a bit commitment scheme where the prover has probability 0 of cheating.

Copies of the paper are available from the authors; the paper will also appear in the proceedings of the 1989 DMMACS workshop.

\section{Definitions and Summary of Results}

Let $A$ and $B$ be two probabalistic polynomial time interactive Turing Machines. Let $\operatorname{conv}\left(R_{A}, R_{B}\right)$ denote the converstion between them, where $R_{A}$ and $R_{B}$ denote their respective random tapes. Each of $A$ and $B$ has a key obtaining algorithm, $K_{A}$ or $K_{B}$; these are polynomial time functions which each party can compute on the conversation and his own coin tosses to yield his estimate of the key. For any instance (i.e. fixed choice of $R_{A}, R_{B}$ )

- Supported in part by NSF grant CCR-87-19689 and DARPA Contract N00014-89-J-1988.

i Supported in part by NSF grant CCR-86-57527 and DARPA Contract N00014-89-J-1988. 
of the protocol we say that $A$ and $B$ have agreed on a key if $K_{A}\left(R_{A}, \operatorname{conv}\left(R_{A}, R_{B}\right)\right)=$ $K_{B}\left(R_{B}, \operatorname{con} v\left(R_{A}, R_{B}\right)\right)$; this common value is the key they have agreed on. There is a security parameter $k$, and for convenience we assume that $\left|R_{A}\right|=\left|R_{B}\right|=k$.

Definition 2.1 A protocol constitutes a secret key exchange ( $S K E$ ) if there is an $\alpha>0$ such that we have

- Agreement: $P_{a g r e e} \geq \alpha$, and on each instance, $A$ and $B$ know whether or not they agreed

- Secrecy: For all probabilistic polynomial time algorithms $E$, for all $d>0$ and all sufficiently large $k$,

$$
P\left(E(C)=K_{A}\left(R_{A}, C\right)=K_{B}\left(R_{B}, C\right): R_{A}, R_{B} \leftarrow\{0,1\}^{k} ; C \leftarrow \operatorname{conv}\left(R_{A}, R_{B}\right)\right) \leq k^{-d} .
$$

Definition 2.2 A protocol constitutes a Weak Secret Key Exchange (weak $S K E$ ) if there is an $\alpha>0$ such that we have

- Agreement: Pagree $\geq \alpha$

- Secrecy: same as in Definition 2.1

It is easy to see that for any conversation $C$ the set of pairs of tapes

$$
\left\{\left(R_{A}, R_{B}\right): \operatorname{conv}\left(R_{A}, R_{B}\right)=C\right\}
$$

is of the form $X \times Y$ (i.e. forms a rectangle). We define the matrix of the conversation $C$ over $X \times Y$ as having as its row $R_{A}$ column $R_{B}$ entry $\left(\left(R_{A}, R_{B}\right) \in X \times Y\right)$

- $K_{A}\left(R_{A}, R_{B}\right)=K_{B}\left(R_{A}, R_{B}\right)$ if these are equal

- some special symbol * otherwise,

Call this matrix $M(C)$. The heart of our results concern properties of this matrix. For example, we show that

Theorem 2.1 For SKE all entries of this matrix are equal (i.e. if $A$ and $B$ agree on $\kappa$ for some pair of random tapes that leads to the conversation $C$, then they agree (and moreover on this same key $\kappa$ ) for all such pairs of random tapes).

Theorem 2.2 For weak SKE, for any conversation $C$ with a "large" area of the matrix not equal to $*$, there is a single key $\kappa$ which covers a corrspondingly large area (i.e. most of the time that $A$ and $B$ agree with conversation $C$, they agree on a particular key $\kappa$ ).

These structural properties imply

Corollary 2.1 SKE implies the existence of a one-way function.

Corollary 2.2 Oblivious transfer implies the existence of a one-way function.

Corollary 2.3 (non-uniform) Weak SKE implies the existence of a one-way function.

Theorems 2.1, 2.2 and the first two corollaries all hold in both the uniform and the nonuniform models. Independently, [Impaliazzo, Luby, (private comunication)], are able to obtain our results for the non-uniform case (corollary 2.3) by viewing Secret Key Exchange as an "identification scheme". Also in a non-uniform model of security, a result of [Naor, Crypto 89] coupled with [Impagliazzo, Levin, Luby STOC 89] implies that one-way functions are enough for bit committment. We prove a stronger result for bit commitment in that: (1) it does not use non-uniform assumptions (2) the scheme is non-interactive (3) the probability of the committer cheating is 0 rather than just very small.

Corollary 2.4 SKE implies bit commitment. 